\title{
Real-World Data on Nonmedical Use of Tramadol from Patients Evaluated for Substance Abuse Treatment in the NAVIPPRO Addiction Severity Index-Multimedia Version (ASI-MV ${ }^{\circledR}$ ) Network
}

\author{
Jody L. Green ${ }^{1}$ (1) $\cdot$ Taryn Dailey-Govoni $^{1} \cdot$ Stephen F. Butler ${ }^{1}$
}

Accepted: 30 September 2020 / Published online: 11 November 2020

(c) The Author(s) 2020

\begin{abstract}
Introduction Drug safety studies regarding comparative risk of different opioid compounds are important as providers and regulatory agencies in the United States continue to balance pain management with an ongoing opioid epidemic.

Objective The aim of this study was to evaluate nonmedical use (NMU) and diversion of tramadol and comparator opioids using real-world data from the Addiction Severity Index-Multimedia Version (ASI-MV ${ }^{\circledR}$ ).

Methods A cross-sectional study design was used to evaluate past 30-day tramadol and comparator opioid NMU among adults assessed for substance abuse treatment using the ASI-MV from 2010 to 2018. Population and drug utilization-adjusted rates were studied, as well as patient characteristics, route of administration, and diversion.

Results Past 30-day NMU of one or more prescription opioid was reported in 125,048 (22.6\%) of ASI-MV assessments (2010-2018); 46.5\% reported oxycodone, $43.2 \%$ hydrocodone, $8.1 \%$ morphine, and 7.2\% tramadol. Male respondents ranged from $43.2 \%$ in the tramadol group to $51.8 \%$ in the oxycodone group. Majority $(\sim 76 \%)$ were Caucasian in all groups, with 86.9\% Caucasian in the morphine group. Prevalence of past 30-day tramadol NMU was significantly lower than that of morphine, oxycodone, and hydrocodone for both population and utilization-adjusted rates. Rate of snorting of tramadol was 4-7 times lower than comparator opioids and injection was 14-34 times lower than morphine and oxycodone. Tramadol was most likely to be obtained via the patient's own prescription while the comparator opioids were more often obtained via dealers or family/friends.

Conclusion Tramadol had a significantly lower rate of NMU than comparator opioids and was less likely to be diverted or used via higher-risk non-oral routes. These findings support previous evaluations by WHO and the United States Drug Enforcement Agency that concluded that tramadol has a low potential for abuse.
\end{abstract}

\section{Introduction}

Drug safety studies from real-world data regarding comparative risk of different opioid compounds are important as providers and regulatory agencies [e.g., the Drug Enforcement Agency (DEA) and the Food and Drug Administration (FDA)] in the United States continue to balance available pain therapies with the ongoing opioid epidemic. Real-world data (data related to patient health status or the delivery of health care) are often used to inform the benefit-risk evaluation of medications in the post-market setting [1]. These data are of particular value when addressing issues not feasible

Jody L. Green

jgreen@inflexxion.com

1 Inflexxion, A Division of Integrated Behavioral Health, Irvine, CA, USA

\section{Key Points}

Utilization of real-world data adds significant value to the post-market benefit-risk evaluation of prescription opioid medications, specifically in relation to direct outcomes of nonmedical use (NMU) and related behaviors.

Comparative rates of NMU between prescription opioid compounds can help providers and patients with pain management decision making, balancing the need for pain therapy with potential risk of NMU.

Compared with other common opioid compounds (oxycodone, hydrocodone, and morphine), tramadol had significantly lower rates of NMU, non-oral routes of administration such as snorting or injecting, and diversion, suggesting a lower abuse potential. 
through clinical trials, studying impact on a population that extends beyond the intended treatment population, and monitoring aberrant behaviors, such as inappropriate use of prescription medications and use of illicit substances.

Tramadol is an opioid agonist indicated in adults for the management of pain severe enough to require an opioid analgesic and for which alternative treatments are inadequate [2]. Tramadol was first approved by the US FDA in 1995 under the trade name Ultram ${ }^{\circledR}$ (Ortho-McNeil Pharmaceutical, Inc., Raritan, NJ, USA), after which other generic and brand tramadol products, including single-entity and combination medications as well as immediate-release (IR) and extendedrelease (ER) formulations, were subsequently approved. To date, the only FDA-approved dosage forms of tramadol medications are tablets and capsules intended to be ingested orally [3], and suppositories. Internationally, tramadol medications are also available in solution for intramuscular or intravenous injection. In 2014, the US DEA placed tramadol into Schedule IV of the Controlled Substance Act based upon the following findings: (1) tramadol has a low potential for abuse relative to the drugs or substances in Schedule III, (2) tramadol has a currently accepted medical use in treatment in the United States (tramadol-containing products are approved for marketing by the FDA to manage moderate to moderately severe pain), and (3) abuse of tramadol may lead to limited physical or psychological dependence relative to other drugs or substances in Schedule III [4]. By definition, Schedule IV controlled substances have a low potential for abuse, while Schedule III controlled substances have a potential for abuse less than substances in Schedules I or II and abuse may lead to moderate or low physical dependence or high psychological dependence, and Schedule II controlled substances have a high potential for abuse which may lead to severe psychological or physical dependence [5]. Determinations are made based upon the drug's acceptable medical use and the abuse or dependency potential. Some examples include heroin (Schedule I); oxycodone and morphine (Schedule II); codeine ( $<90 \mathrm{mg}$ per dosage unit) and anabolic steroids (Schedule III); and tramadol, alprazolam, and zolpidem (Schedule IV).

Since the DEA's final rule in 2014, the abuse potential of tramadol has been further questioned based upon research using prescribing patterns as a surrogate for inappropriate use. An observational study of administrative claims data investigated the risk of prolonged opioid use in patients receiving tramadol compared with other short-acting opioids, concluding that post-surgical patients that received tramadol alone had similar to somewhat higher risks of prolonged opioid use compared with those receiving other short-acting opioids [6]. Another study noted the increase in tramadol prescriptions dispensed following the rescheduling of hydrocodone-containing products from Schedule III to Schedule II in 2014, suggesting that mere drug utilization is a surrogate for inappropriate use and increased risk [7]. These, among other studies of mortality rates not specific to misuse or abuse $[8,9]$, were cited in a Citizen Petition submitted to the DEA and FDA in November 2019 that requested the rescheduling of tramadol and like compounds from Schedule IV to Schedule II due to its purported high level of abuse, with use potentially leading to severe psychological or physical dependence [10]. The studies cited in the Citizen Petition conflict with the DEA's final rule, multiple reviews conducted by the World Health Organization [11], and with the majority of previously published literature on tramadol safety and abuse potential, including a recent systematic review of laboratory evidence [12]. The conflicting data are likely due to the data sources, outcome measures, and methodologies employed as well as confounders that prevent evaluation of a causal relationship. The studies that suggest increasing rates of negative consequences with tramadol use include contemporary data but also have limitations in relation to an evaluation of abuse potential, including the use of surrogate measures for actual consumption and inappropriate use. This is a well-known limitation of drug utilization and claims data that involves using a substitute measure in place of a direct measure of outcomes or behavior. Use of these data often assumes that prescribing or dispensing is equivalent to actual use of a product or appropriateness of use, that all medications prescribed or dispensed at the pharmacy were consumed, and that only medications prescribed or dispensed were accessible (does not capture medications obtained from other sources or illicit drug use) [13]. Additional limitations include the use of non-opioid-specific outcomes (e.g., all-cause mortality rather than opioid-related mortality) and inability to measure real-world opioid use or drug-related behaviors such as route of administration and diversion.

Data sources that capture actual use and drug-related behaviors, including the Addiction Severity Index-Multimedia Version (ASI-MV ${ }^{\circledR}$, Inflexxion, a division of Integrated Behavioral Health, Irvin, CA, USA), may provide more accurate insights into nonmedical use (NMU) of prescription opioid medications. ASI-MV data have been previously used to evaluate NMU prevalence of specific opioid products or compounds [14-16] and effectiveness of interventions such as abuse deterrent formulations [17].

An accurate understanding of abuse potential of different analgesic therapies is important for both providers and patients as they balance pain management with associated risks of misuse, abuse or diversion. The objective of this study was to evaluate NMU and diversion of tramadol and comparator opioids in the United States. Prevalence rates, patient characteristics, route of administration, and source of drug procurement were studied using real-world epidemiological data from the National Addictions Vigilance 
Intervention and Prevention Program (NAVIPPRO ${ }^{\circledR}$ ) ASIMV network.

\section{Methods}

This is a cross-sectional surveillance study design used to examine past 30-day tramadol NMU and comparator Schedule II prescription opioid NMU (i.e., morphine, oxycodone, and hydrocodone) among adults aged 18 years or older assessed for substance abuse problems and treatment planning using the ASI-MV® clinical assessment tool from 01 January 2010 through 31 December 2018. Individuals in substance abuse treatment comprise a sentinel population in which to detect emerging patterns of prescription drug NMU and illicit drug abuse. For the purposes of this study, NMU was defined as (1) use of a prescription medication that was not prescribed to the patient by a healthcare provider, (2) use in ways other than prescribed, or (3) use for reasons other than prescribed. The NMU definition is determined by behavior rather than intent, hence it includes both misuse for therapeutic intent as well as abuse for psychotropic effects or to get high.

The ASI-MV is a proprietary clinical assessment tool used in standard clinical work flow to evaluate adults for substance abuse and treatment planning. This network includes locations from across the United States; however, it is a convenience sample of sites that license the tool for clinical practice and is not considered nationally representative. The ASI-MV is a self-administered, structured, computerized interview based upon the original Addiction Severity Index, which requires an in-person interview. The ASI-MV collects patient-reported demographics and other characteristics as well as generates composite scores and severity scores for seven domains (medical, employment, alcohol, drug, legal, family/social, and psychiatric) that inform treatment planning. The ASI-MV was developed with support from the National Institute on Drug Abuse (NIDA) and has good test-retest reliability, discriminant validity, and criterion validity $[18,19]$.

The characteristics of the study groups were summarized, including sex, age, race/ethnicity, census region of ASI-MV site where the assessment was completed, and drug severity rating. In the ASI-MV assessment, responses to several questions regarding substance use are used to calculate the drug severity rating, which ranges from 0 to $9[18,19]$. Interpretation of the drug severity rating is as follows: $0-1$, no real problem; $2-3$, a slight problem; $4-5$, a moderate problem; 6-7, a severe problem; and 8-9, an extreme problem. Note that the rating cannot be calculated if certain questions are not answered, and in these cases the drug severity rating will be missing.
Using decision tree logic that allows the ASI-MV to simulate an interviewer, respondents are guided through questions about use of pharmaceutical substances. The ASI-MV contains product-specific questions about general use, NMU, routes of administration, and diversion (measured as sources of drug procurement; e.g. dealer, friends/ family, own physician). Specific opioid medications are identified by presenting screens with pharmaceutical opioid product names (trade, generic, and slang names) and pictures, along with audio, so that respondents are better able to differentiate between various products that they report having used. When a respondent has completed the clinical assessment at the treatment site, individual-level data are de-identified and electronically uploaded to a central server where they are available for analysis.

The primary outcome of this study was past 30-day tramadol NMU (alone or in combination with other prescription opioids) and comparator opioids (morphine, oxycodone, and hydrocodone), overall and by specific routes of administration (i.e., oral, snort, smoke, and inject).

The primary opioid of interest for this analysis was tramadol. Both IR and ER tramadol products were included. Tramadol users were further stratified into the following two groups to study tramadol NMU alone versus tramadol NMU as part of a more complex polysubstance use pattern: (a) those who only reported past 30-day tramadol NMU, and (b) those who reported past 30-day tramadol NMU plus past 30-day NMU of at least one other prescription opioid compound monitored in the ASI-MV. Comparator opioids included solid oral dosage formulations (e.g. tablets, capsules) of IR and ER formulations of morphine, oxycodone, and hydrocodone, all of which are Schedule II controlled substances. The comparator opioids oxycodone and hydrocodone were chosen as those most readily available as they comprise the majority of the prescriptions dispensed from outpatient pharmacies. All comparators are similar to tramadol in that they are primarily IR products but are also available as ER formulations. Also-like tramadol-morphine, oxycodone, and hydrocodone products are primarily generic products and available at relatively low cost. Morphine is also a common comparator in published studies on abuse potential [12]. Past 30-day NMU of the comparator opioid compounds included mentions of such products, alone or in combination with other opioid compounds. Hence, the following groups were studied: (1) any tramadol, (1a) tramadol only, (1b) tramadol plus any other prescription opioid compound, (2) morphine, (3) oxycodone, and (4) hydrocodone. Any tramadol and the comparator groups were not mutually exclusive as past 30-day NMU of multiple drug types could be reported.

Annualized NMU was assessed during the aggregate study period using the following approaches: (1) rate among all ASI-MV assessments completed during the study period 
(i.e., the total study population), (2) rate adjusted by the number of prescriptions dispensed, and (3) rate adjusted by the number of solid oral dosage units (e.g., tablets, capsules, caplets) dispensed. The $95 \%$ confidence intervals for NMU rates were calculated using a binomial distribution or Poisson distribution (i.e., in instances in which the number of cases was $<30$ ). Significant differences in rates between comparator groups were defined as instances where $95 \%$ confidence intervals did not overlap. Prescriptions and 'units dispensed' data were obtained from IQVIA (Danbury, CT, USA) as derived from their National Prescription Audit ${ }^{\mathrm{TM}}$ (NPA), which estimates prescriptions and units dispensed from the universe of retail, standard mail service, specialty mail service, and long-term care pharmacies. Only states with sites that contributed at least one assessment to the ASI-MV dataset during a particular calendar year during the 9-year study period were included in the prescription-adjusted analyses. The frequency and proportion of past 30-day NMU via oral administration, snorting, smoking, and injection are also described. The reported routes of administration within each group were not mutually exclusive as one patient could have reported use via more than one route. Patients were included in the counts and rates for each route they reported within that product group.

\section{Results}

During 2010 through 2018, a total of 554,265 ASI-MV assessments were submitted from 1267 sites located in 48 states throughout the United States. Of these assessments, 125,048 (22.6\%) reported past 30-day NMU of at least one prescription opioid medication. The greatest number of past 30-day NMU reports were observed for oxycodone ( $n=58,115 ; 46.5 \%)$, followed by hydrocodone $(n=53,992$; $43.2 \%)$, morphine $(n=10,180 ; 8.1 \%)$, and any tramadol $(n=8942 ; 7.2 \%)$ (Table 1$)$. The sum of these study groups exceeds the total number of assessments that included NMU of at least one prescription opioid as more than one prescription opioid could have been reported for each assessment; hence, they are not mutually exclusive. The mean number of prescription opioid products reported for past 30-day NMU were 6.2 (SD 5.23) for the 'any tramadol' group, 7.8 (SD 5.47) for the morphine group, 4.9 (SD 3.98) for the oxycodone group, and 4.5 (SD 4.11) for the hydrocodone group (data not shown). The study group of past 30-day NMU of any tramadol comprised two mutually exclusive subgroups, $1105(12.4 \%)$ reports of tramadol NMU only (no other prescription opioids were reported for past 30-day NMU) and 7837 (87.6\%) reports of tramadol NMU plus NMU of at least one other prescription opioid compound. The mean number of prescription opioid products reported for the subgroup of tramadol NMU plus NMU of at least one other opioid was 6.9 (SD 5.2).

Among those who reported past 30-day NMU of tramadol or comparators, the proportion of male respondents ranged from $43.2 \%$ in the 'any tramadol' group to $51.8 \%$ in the oxycodone group (Table 1). Those who reported past 30-day NMU of any tramadol were more likely to be older (age $35+$ years) than those who reported morphine and oxycodone particularly. While the majority of all study groups were Caucasian, the morphine group included $86.9 \%$ Caucasian, which was about $10 \%$ higher than the oxycodone, hydrocodone, and 'any tramadol' groups. Geographical differences were also detected; the distribution of past 30-day NMU of any tramadol was 10-44\% higher in the Midwest than the comparator groups and 9-24\% lower in the South than the comparator drug groups.

The highest drug severity ratings, represented by the sum of those who scored as a severe or extreme problem, were observed among nonmedical users of morphine (84.8\%), followed by oxycodone (74.6\%), any tramadol (73.0\%), and then hydrocodone (68.5\%) (Table 1). Cases of past 30-day NMU of tramadol only had the lowest drug severity ratings (38.6\% severe or extreme problem) compared with those that reported past 30-day NMU of tramadol plus at least one other prescription opioid compound $(77.8 \%$ severe or extreme problem) or any other comparator drug group.

The prevalence of past 30-day NMU of any tramadol (1.61 cases per 100 ASI-MV assessments) was significantly lower than the prevalence of past 30-day NMU of morphine (1.84 cases per 100 ASI-MV assessments), oxycodone (10.49 cases per 100 ASI-MV assessments), or hydrocodone (9.74 cases per 100 ASI-MV assessments) (Table 2). The greatest number of prescriptions and units dispensed among states contributing assessment data to the ASI-MV network during the study period were observed for hydrocodone, followed by oxycodone, tramadol, and then morphine. Utilization-adjusted rates of NMU (i.e., per 100,000 prescriptions dispensed; per 10,000,000 units dispensed) of any tramadol were also significantly lower than those observed for morphine, oxycodone, or hydrocodone. Specifically, any tramadol was 2-3 times lower than hydrocodone, 4-5 times lower than oxycodone, and 5-6 times lower than morphine. 'Tramadol alone' rates were the lowest by far for both population and drug utilization-adjusted rates.

Annual rates showed a significant decrease during the study period in NMU per 100 ASI-MV assessments for oxycodone and hydrocodone while NMU rates for tramadol and morphine remained relatively stable (Fig. 1). When adjusted for prescriptions dispensed or units dispensed, significant decreases in NMU during the study period were found for tramadol, morphine, and oxycodone while NMU of hydrocodone significantly increased, particularly in 2015 and 2016. 
Table 1 Patient demographics and geographic distribution of ASI-MV assessments by study group (01 January 2010 to 31 December 2018)

\begin{tabular}{|c|c|c|c|c|c|c|}
\hline & Any tramadol & Tramadol only & $\begin{array}{l}\text { Tramadol } \\
\text { PLUS other } \\
\text { opioid }^{\mathrm{b}}\end{array}$ & Morphine & Oxycodone & Hydrocodone \\
\hline$N^{\mathrm{a}}$ (\% of prescription opioid NMU cases) & $8942(7.2 \%)$ & 1105 & 7837 & $10,180(8.1 \%)$ & $58,115(46.5)$ & $53,992(43.2 \%)$ \\
\hline \multicolumn{7}{|l|}{ Sex } \\
\hline Male & $3865(43.2)$ & $549(49.7)$ & $3,316(42.3)$ & $4850(47.6)$ & $30,079(51.8)$ & $26,936(49.9)$ \\
\hline Female & $5076(56.8)$ & $556(50.3)$ & $4,520(57.7)$ & $5329(52.3)$ & $28,032(48.2)$ & $27,046(50.1)$ \\
\hline Unknown/not reported & $1(<0.01)$ & $O(<0.01)$ & $1(<0.01)$ & $1(<0.01)$ & ) $\quad 4(<0.01)$ & $10(<0.01)$ \\
\hline \multicolumn{7}{|l|}{ Age (years) } \\
\hline$<21$ & $483(5.4)$ & $44(4.0)$ & $439(5.6)$ & $594(5.8)$ & $4132(7.1)$ & $3487(6.5)$ \\
\hline $21-34$ & $4694(52.5)$ & $387(35.0)$ & $4307(55.0)$ & $6173(60.6)$ & $34,384(59.2)$ & $30,559(56.6)$ \\
\hline $35-54$ & $3301(36.9)$ & $535(48.4)$ & $2766(35.3)$ & $3095(30.4)$ & $17,627(30.3)$ & $17,869(33.1)$ \\
\hline $55+$ & $464(5.2)$ & $139(12.6)$ & $325(4.1)$ & $318(3.1)$ & $1972(3.4)$ & $2,077(3.8)$ \\
\hline \multicolumn{7}{|l|}{ Race/ethnicity } \\
\hline Caucasian & $6853(76.6)$ & $733(66.3)$ & $6120(78.1)$ & $8849(86.9)$ & $43,861(75.5)$ & $41,193(76.3)$ \\
\hline African American & $807(9.0)$ & $155(14.0)$ & $652(8.3)$ & $352(3.5)$ & $6246(10.7)$ & $4964(9.2)$ \\
\hline Hispanic/Latino & $736(8.2)$ & $110(10.0)$ & $626(8.0)$ & $560(5.5)$ & $5511(9.5)$ & $5134(9.5)$ \\
\hline American Indian or Alaska Natives & $338(3.8)$ & $83(7.5)$ & $255(3.3)$ & $238(2.3)$ & $1484(2.6)$ & $1615(3.0)$ \\
\hline Asian & $37(0.4)$ & $4(0.4)$ & $33(0.4)$ & $31(0.3)$ & $269(0.5)$ & $260(0.5)$ \\
\hline Other & $171(1.9)$ & $20(1.8)$ & $151(1.9)$ & $150(1.5)$ & $743(1.3)$ & $825(1.5)$ \\
\hline \multicolumn{7}{|l|}{ US Census region } \\
\hline South & $4313(48.2)$ & $507(45.9)$ & $3806(48.6)$ & $5689(55.9)$ & $34,847(60.0)$ & $28,333(52.5)$ \\
\hline West & $1184(13.2)$ & $199(18.0)$ & $985(12.6)$ & 873 (8.6) & $8690(15.0)$ & $9260(17.2)$ \\
\hline Midwest & $3117(34.9)$ & $372(33.7)$ & $2745(35.0)$ & $3193(31.4)$ & $11,322(19.5)$ & $14,629(27.1)$ \\
\hline Northeast & $328(3.7)$ & $27(2.4)$ & $301(3.8)$ & $425(4.2)$ & $3256(5.6)$ & $1770(3.3)$ \\
\hline \multicolumn{7}{|l|}{ Drug severity rating } \\
\hline No real problem $(0-1)$ & 705 (7.9) & $285(25.8)$ & $420(5.4)$ & $227(2.2)$ & $4104(7.1)$ & $6038(11.2)$ \\
\hline Slight problem (2-3) & $465(5.2)$ & $164(14.8)$ & $301(3.8)$ & $220(2.2)$ & $2576(4.4)$ & $3050(5.6)$ \\
\hline Moderate problem (4-5) & 799 (8.9) & $162(14.7)$ & $637(8.1)$ & $617(6.1)$ & $5587(9.6)$ & $5687(10.5)$ \\
\hline Severe problem (6-7) & $2700(30.2)$ & $284(25.7)$ & $2416(30.8)$ & $3039(29.9)$ & $19,899(34.2)$ & $17,198(31.9)$ \\
\hline Extreme problem (8-9) & $3824(42.8)$ & $142(12.9)$ & $3682(47.0)$ & $5596(55.0)$ & $23,429(40.3)$ & $19,774(36.6)$ \\
\hline Missing/no response & $449(5.0)$ & $68(6.2)$ & $381(4.9)$ & $481(4.7)$ & $2520(4.3)$ & $2245(4.2)$ \\
\hline
\end{tabular}

ASI-MV Addiction Severity Index-Multimedia Version, $N M U$ nonmedical use

${ }^{a}$ Respondents could have reported more than one prescription opioid used in past 30 days for NMU, hence the comparator groups are not mutually exclusive

b'Tramadol only' and 'Tramadol PLUS other opioid' are mutually exclusive subsets of the 'Any tramadol' group

With respect to reported routes of administration, the majority of past 30-day NMU of any tramadol was via oral routes of administration $(92.0 \%)$, while $6.3 \%$ reported snorting, $0.5 \%$ reported smoking, and $1.5 \%$ reported injection (Fig. 2a). The proportion of past 30-day NMU of any tramadol via snorting was four times lower than morphine, seven times lower than oxycodone, and four times lower than hydrocodone. The proportion of past 30-day NMU of any tramadol via injecting was 34 times lower than morphine and 14 times lower than oxycodone. The proportions of past 30-day NMU via injection for any tramadol and hydrocodone were similar. A greater proportion of the tramadol plus other prescription opioid compounds group reported snorting (7.1\% versus $0.4 \%)$ and smoking $(0.5 \%$ versus $0.0 \%$ ) compared with those who indicated NMU of tramadol only. When evaluated per number of units dispensed, past 30-day NMU of tramadol via oral routes was reported at a rate of 0.40 cases per 1,000,000 units dispensed, followed by snorting ( 0.03 cases per $1,000,000$ units dispensed), injection ( 0.01 cases per $1,000,000$ units dispensed), and then smoking (0.00 cases per 1,000,000 units dispensed) (Fig. 2b). Relative to the comparator opioids, tramadol had the lowest reported rate of NMU per units dispensed via oral routes, snorting, smoking, and injection. Oxycodone had the highest rate of NMU via oral routes (14.31 cases per 10,000,000 units dispensed), 
Table 2 Population and drug utilization-adjusted rates of NMU for tramadol and comparator opioid products (01 January 2010 to 31 December 2018)

\begin{tabular}{|c|c|c|c|c|c|c|}
\hline Past 30-day NMU & Total NMU cases & $\begin{array}{l}\text { Rate/100 ASI-MV } \\
\text { assessments (95\% } \\
\text { CI) }\end{array}$ & $\begin{array}{l}\text { Total pre- } \\
\text { scriptions } \\
\text { dispensed }^{\mathrm{b}}\end{array}$ & $\begin{array}{l}\text { Rate } / 100,000 \text { pre- } \\
\text { scriptions dispensed } \\
(95 \% \mathrm{CI})\end{array}$ & $\begin{array}{l}\text { Total units } \\
\text { dispensed }^{c}\end{array}$ & $\begin{array}{l}\text { Rate } / 10,000,000 \\
\text { units dispensed (95\% } \\
\text { CI) }\end{array}$ \\
\hline Tramadol & 8942 & $1.61(1.58-1.65)$ & $275,526,568$ & $3.25(3.18-3.31)$ & $20,640,131,569$ & $4.33(4.24-4.42)$ \\
\hline Tramadol only & 1105 & $0.20(0.19-0.21)$ & $275,526,568$ & $0.40(0.38-0.42)$ & $20,640,131,569$ & $0.54(0.50-0.57)$ \\
\hline $\begin{array}{l}\text { Tramadol plus any } \\
\text { other Rx opioid }\end{array}$ & 7837 & $1.41(1.38-1.45)$ & $275,526,568$ & $2.84(2.78-2.91)$ & $20,640,131,569$ & $3.80(3.71-3.88)$ \\
\hline Morphine & 10,180 & $1.84(1.80-1.87)$ & $55,582,130$ & $18.32(17.96-18.67)$ & $4,003,584,090$ & $25.43(24.93-25.92)$ \\
\hline Oxycodone & 58,115 & $10.49(10.40-10.57)$ & $405,895,655$ & $14.32(14.20-14.43)$ & $30,141,631,493$ & $19.28(19.12-19.44)$ \\
\hline Hydrocodone & 53,992 & $9.74(9.66-9.82)$ & $755,796,049$ & $7.14(7.08-7.20)$ & $44,189,293,091$ & $12.22(12.12-12.32)$ \\
\hline
\end{tabular}

ASI-MV Addiction Severity Index-Multimedia Version, $C I$ confidence interval, $N M U$ nonmedical use, $R x$ prescription

${ }^{a}$ pioid categories are not mutually exclusive in that one patient could have reported more than one product

${ }^{\mathrm{b}}$ The total prescriptions dispensed included projected prescriptions dispensed in states participating in the ASI-MV network during the study period

${ }^{\mathrm{c}}$ The total number of units dispensed included projected solid oral dosage units (e.g. tablets, capsules, caplets) dispensed in states participating in the ASI-MV network during the study period

${ }^{\mathrm{d}}$ This category represents past 30-day NMU of tramadol and past 30-day NMU of at least one other prescription opioid compound monitored in the ASI-MV

snorting (8.39 cases per 10,000,000 units dispensed), and smoking (1.12 cases per 10,000,000 units dispensed), while morphine had the highest rate of NMU via injection (13.45 cases per 10,000,000 units dispensed).

The patient's own prescription was the most common source of drug for those who reported past 30-day tramadol NMU (46.6\% any tramadol; $68.9 \%$ tramadol alone; $43.5 \%$ tramadol plus any other prescription opioid) and was more likely to be procured via the patient's own prescription than the comparator opioids (tramadol $46.6 \%$, morphine $11.1 \%$, oxycodone $25.8 \%$, and hydrocodone $38.6 \%$ ) (Fig. 3). The primary source for morphine was a dealer $(47.3 \%)$ followed by family/friend $(38.7 \%)$. The primary sources for oxycodone were family/friend (42.7\%) and dealer (42.8\%), and the primary source for hydrocodone was family/friend $(42.6 \%)$ followed by the patient's own prescription (38.6\%). These data illustrate a lower rate of diversion for tramadol NMU than for comparator opioid NMU.

\section{Discussion}

This study illustrates the differences in real-world NMU and related behaviors for tramadol compared with morphine, oxycodone, and hydrocodone from the ASI-MV network of adults assessed for substance abuse problems and treatment planning. During 2010 through 2018, the population and drug utilization-adjusted rates of past 30-day NMU of tramadol (including NMU of tramadol only and NMU of tramadol plus at least one other prescription opioid compound) were significantly lower than the rates of past 30-day NMU of morphine, oxycodone, or hydrocodone. Depending on the method of estimation, NMU rates of comparator opioids were up to 6.5-fold higher than NMU rates of tramadol. The relative lower rate of tramadol NMU and tramadol diversion found in this study supports that of previously published real-world data in the post-market setting from independent data sources $[12,16$, 20-24]. In one of the most recent studies [20], drug utilization-adjusted rates of tramadol intentional abuse exposures collected by poison centers were the second lowest of the opioids studied (hydrocodone 0.020 exposures/100,000 units dispensed, tramadol 0.022 exposures/100,000 units dispensed, morphine 0.052 exposures/100,000 units dispensed, and oxycodone 0.166 exposures/100,000 units dispensed); tramadol drug diversion rates were significantly lower than all other opioids studied with the exception of tapentadol, which had a similar rate (tramadol 0.047 exposures/100,000 units dispensed, hydrocodone 0.132 exposures/100,000 units dispensed, oxycodone 0.272 exposures/100,000 units dispensed, and morphine 0.274 exposures/100,000 units dispensed); and past 30-day 'to get high' rates of tramadol from a substance abuse treatment center program were significantly lower than all other opioids studied (tramadol 0.036 exposures/100,000 units dispensed, hydrocodone 0.166 exposures/100,000 units dispensed, oxycodone 0.337 exposures/100,000 units dispensed, and morphine 1.085 exposures/100,000 units dispensed).

In this study, tramadol NMU was predominately via oral routes of administration, though snorting, smoking, and injection were also reported to a lesser extent. Drug 
Fig. 1 Annual population and drug utilization-adjusted rates of NMU for tramadol and comparator opioid product NMU (01 January 2010 to 31 December 2018). ASI-MV Addiction Severity Index-Multimedia Version, $N M U$ nonmedical use, $R x$ prescription

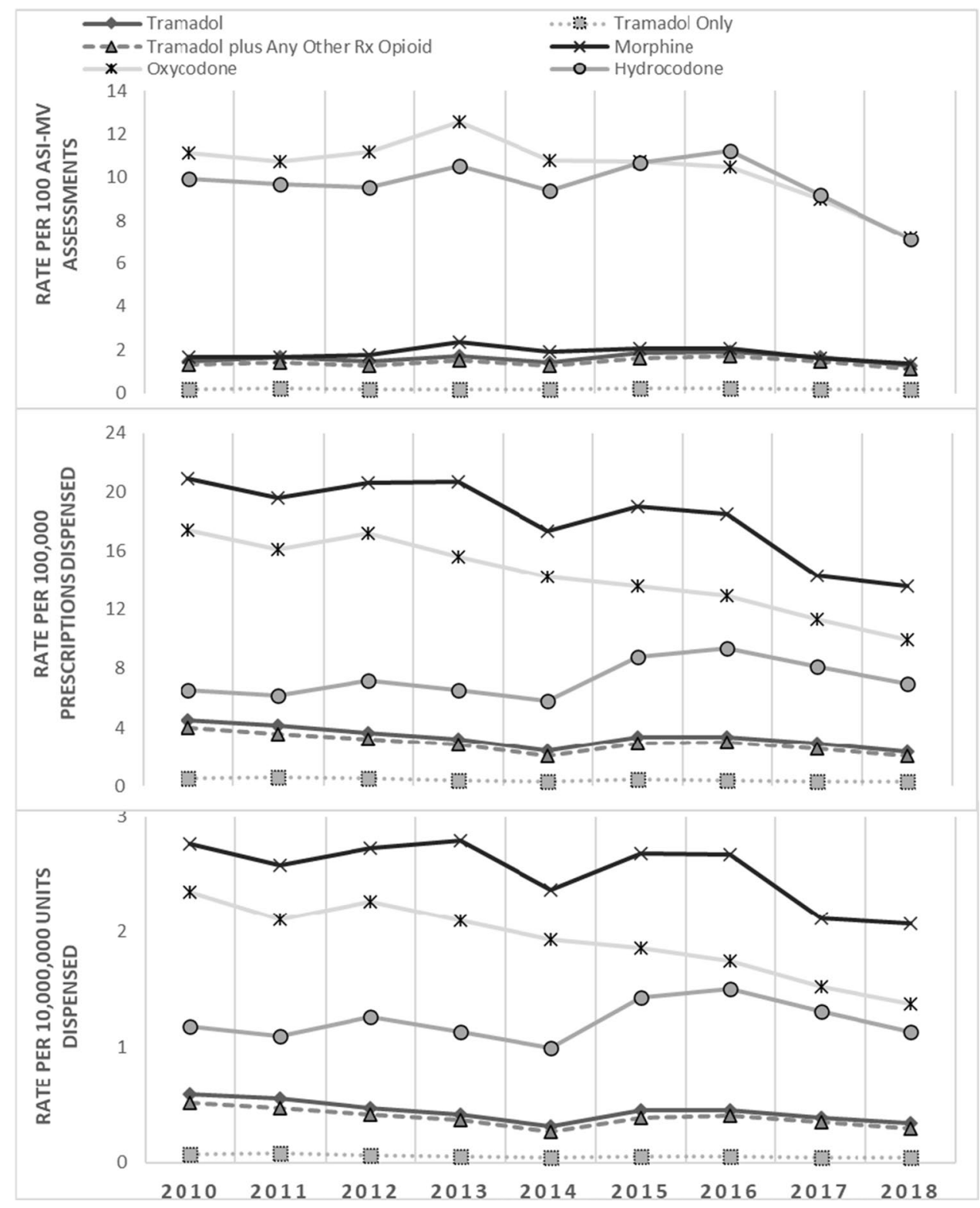

utilization-adjusted rates suggest a significantly lower risk of non-oral routes of administration among those who reported past 30-day NMU of tramadol than among those who reported past 30-day NMU of morphine, oxycodone, or hydrocodone. Snorting of tramadol was reported at a rate 4-7 times lower than morphine, oxycodone, and hydrocodone and injecting tramadol was reported at a rate 14-34 times lower than morphine and oxycodone injection. These data are consistent with a systematic review of the laboratory evidence regarding the abuse potential of tramadol from which the authors concluded that the greatest risk of tramadol abuse was via oral routes of administration and the likelihood of tramadol abuse decreased when administered parentally [12]. Non-oral routes of administration for opioid NMU have been associated with more severe medical outcomes. In a study of poison center exposures, the relative risk of a life-threatening outcome or death following a non-oral opioid exposure was 2.43 (95\% CI 1.97-2.99) compared with an oral opioid exposure [25]. Hence, prescription opioids with a lower likelihood of non-oral NMU are expected to carry lower risk of significant medical outcomes in abuse-related exposures.

Individuals who reported past 30-day NMU of tramadol were most often polysubstance users; $87.6 \%$ reported $\mathrm{NMU}$ of at least one other prescription opioid compound in the past 30 days. Only $12.4 \%$ of those who reported past 30 -day tramadol NMU reporting only using tramadol. The characteristics of tramadol subgroups suggest differences 
Fig. 2 Proportion (a) and drug utilization-adjusted rates (b) of past 30-day NMU of tramadol and comparators via specific routes of administration within ASI-MV network (01 January 2010 to 31 December 2018). ASI-MV Addiction Severity Index-Multimedia Version, $N M U$ nonmedical use, $\mathrm{Rx}$ prescription, error bars $95 \%$ confidence interval. Note: Opioid compounds (tramadol, morphine, oxycodone, and hydrocodone) are not mutually exclusive in that one patient could have reported more than one product. However, the subgroups of tramadol (i.e., tramadol only, and tramadol plus any other prescription opioid) are mutually exclusive from one another. The tramadol plus any other prescription opioid subgroup represents past 30-day NMU of tramadol and past 30-day NMU of at least one other prescription opioid compound monitored in the ASI-MV. Note: Route of administration response options are not mutually exclusive in that multiple ROAs can be selected for a single product

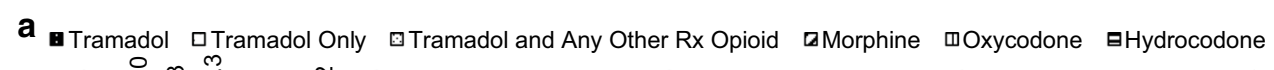

ORAL

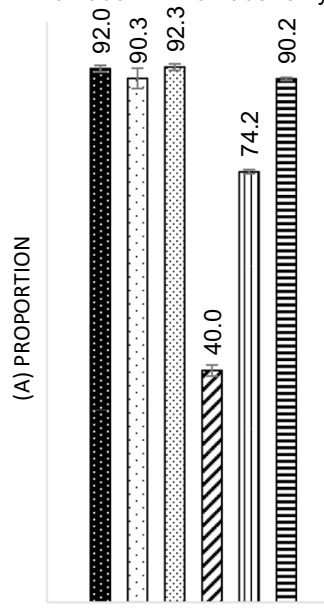

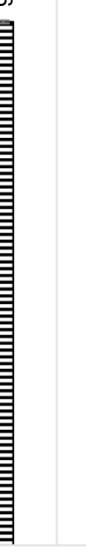

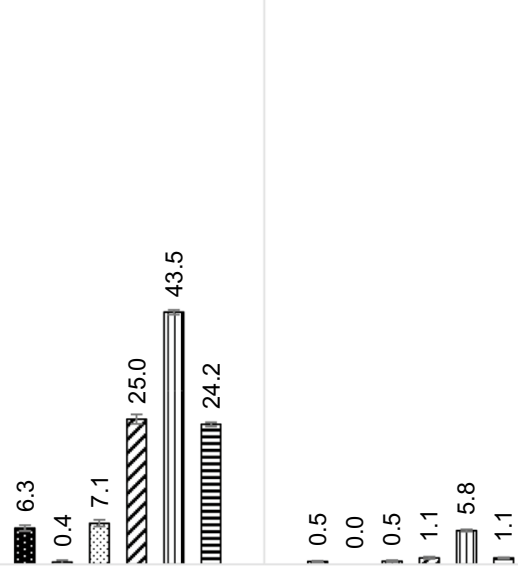

SMOKE

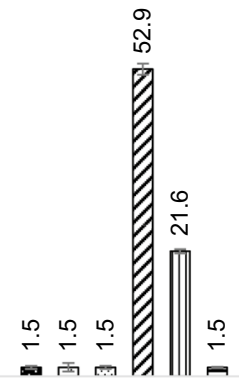

INJECT

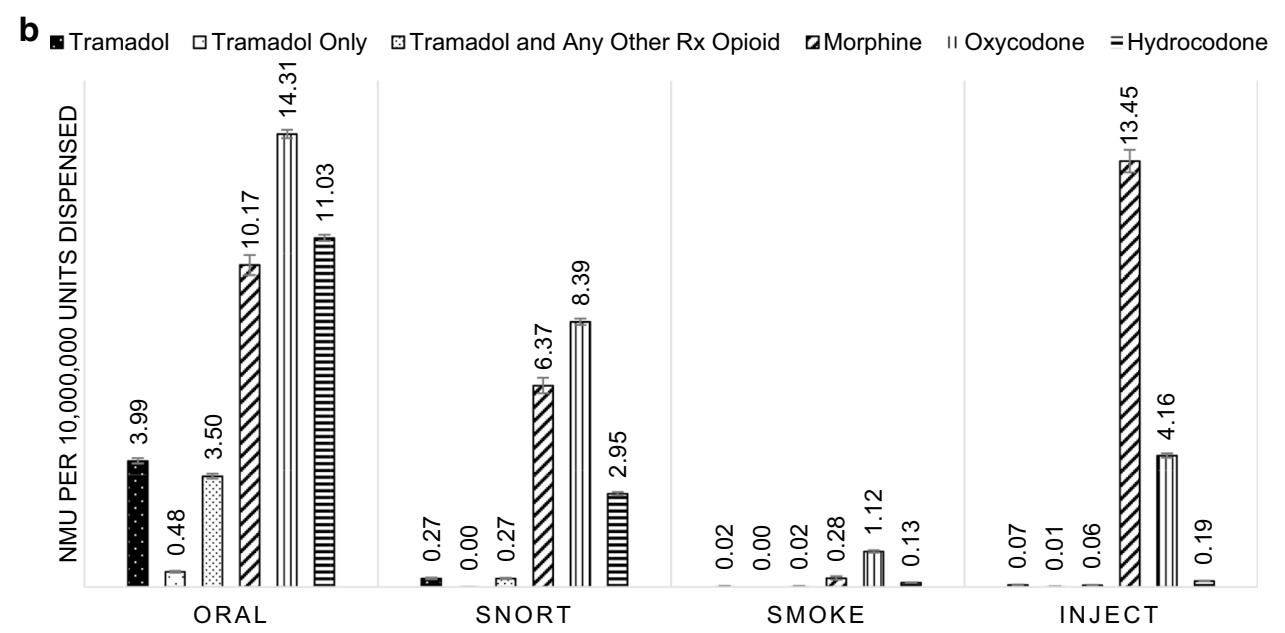

between those who report past 30-day NMU of tramadol alone versus past 30-day NMU of tramadol plus at least one other prescription opioid. Those who reported use of other prescription opioid compounds were more likely to engage in snorting, procure medications via illicit sources, and have a severe or extreme problem rating (drug severity score). Notably, those who reported past 30-day NMU of tramadol alone were more likely to be older (35 years and older) and obtain tramadol through their own prescription $(68.9 \%)$. Emerging trends related to polysubstance use have recently been studied in a similar population of individuals seeking substance abuse treatment [26]. From 2011 to 2018 , the proportion of the population that reported abuse of prescription opioids only has dramatically decreased while polysubstance abuse has increased, including abuse of non-opioid prescription drugs and other illicit drugs. These patterns suggest a more complex syndrome of substance use and complicate evaluation of specific prescription compounds.

\subsection{Limitations}

As with most real-world data sources, limitations related to reliance on self-report of aberrant behaviors and recollection of specific products used and in what manner, apply. While these data are collected during clinical practice at locations throughout the US, they are not nationally representative. However, they provide a valuable source of drug NMU among a sentinel, enriched population of adult prescription drug users within the ASI-MV treatment center population. It is unknown if these results are generalizable to the larger population of individuals seeking substance abuse treatment. The strengths of this study include the collection of NMUspecific outcomes, including reports of past 30-day NMU for tramadol and comparator opioids, source of drug procurement (drug diversion), and route of administration. This study also uses both population and drug utilization-adjusted rates of NMU, as it is necessary to account for the extent that each opioid is available for NMU in the community [27]. 
Fig. 3 Diversion (source of drug procurement) by individuals within the ASI-MV network who reported past 30-day NMU of tramadol and comparator opioids (01 January 2010 to 31 December 2018). ASI-MV Addiction Severity Index-Multimedia Version, $N M U$ nonmedical use, $R x$ prescription. Note: Opioid compounds (tramadol, morphine, oxycodone, and hydrocodone) are not mutually exclusive in that one patient could have reported more than one product. However, the subgroups of tramadol (i.e., tramadol only, and tramadol plus any other prescription opioid) are mutually exclusive from one another. The tramadol plus any other prescription opioid subgroup represents past 30-day NMU of tramadol and past 30-day NMU of at least one other prescription opioid compound monitored in the ASI-MV
- Tramadol aTramadol Only $₫$ Tramadol plus any Other Rx Opioid aMorphine mOxycodone aHydrocodone

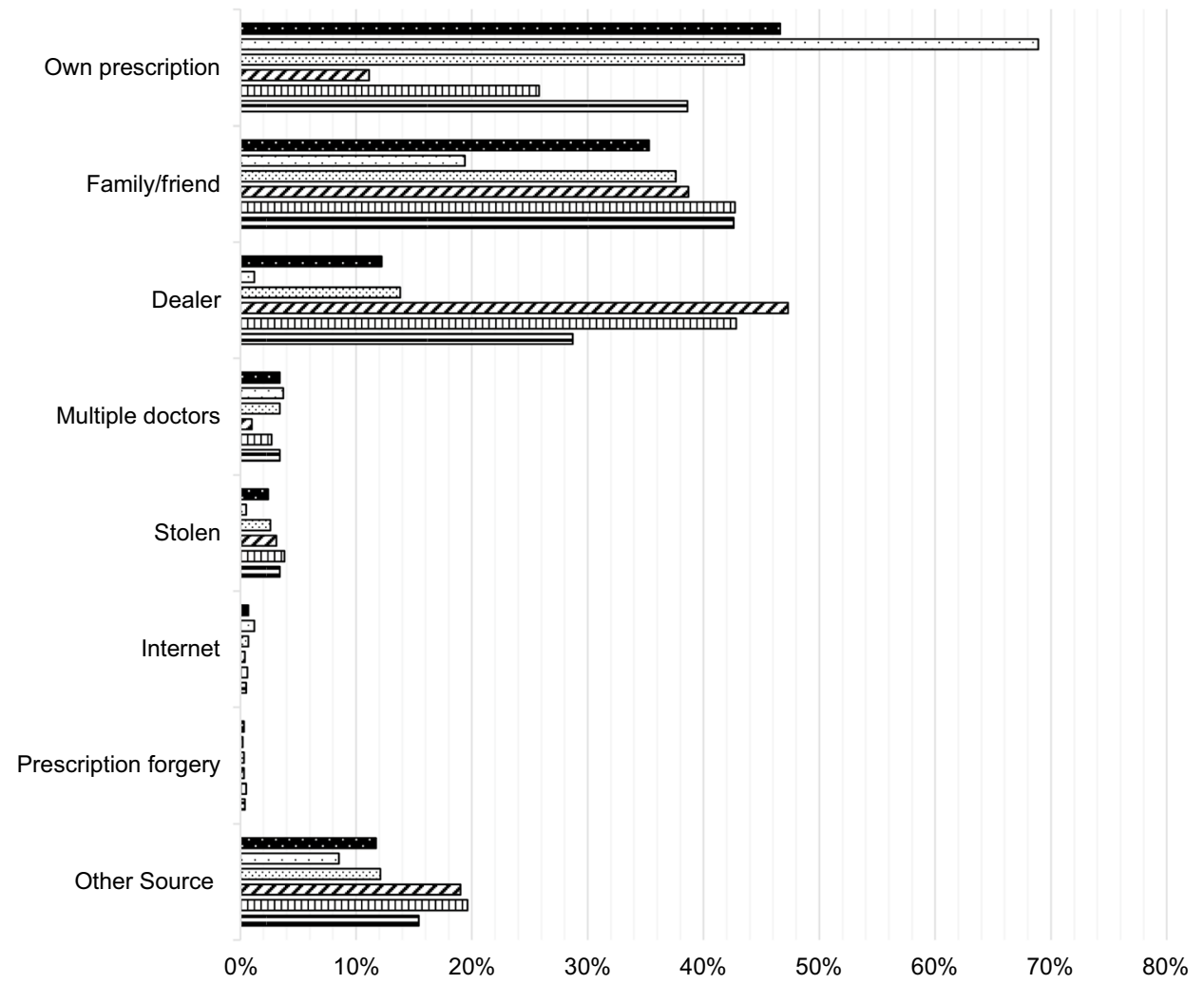

\section{Conclusion}

The real-world data evaluated in this study suggests that tramadol has a significantly lower rate of NMU than morphine, oxycodone, or hydrocodone and is less likely to be diverted or used via higher-risk non-oral routes among adults assessed for substance abuse problems and treatment planning using the ASI-MV clinical assessment tool. Understanding abuse potential of different analgesic therapies is important for providers and patients as they balance the benefits of pain therapy with the potential risks associated with NMU, particularly when used via non-oral routes of administration. The most recent epidemiological evidence, including findings from this study, support earlier evaluations by the WHO and DEA that concluded that tramadol has a low potential for abuse and is appropriately designated as a Schedule IV drug.

\section{Declarations}

Funding Funding for this research was provided by Avenue Therapeutics.

Conflict of Interest TDG and JLG are employees of and SFB is a consultant to Inflexxion, a division of Integrated Behavioral Health. Inflexxion contracts with government agencies as well as multiple pharmaceutical companies that market some of the products included in the study groups evaluated for this article. Although the sponsor was involved in reviewing the content of this article, all data collection, analysis, and ultimate data interpretation were made by the authors without sponsor influence.

Ethics Approval This study uses deidentified data collected during routine clinical workflow, hence it is not human subject research and is exempt from requirements for institutional review board review.

Consent to Participate Not applicable.

Consent for Publication Not applicable.

Availability of Data and Material The datasets generated and analyzed during the current study are not publicly available as they are proprietary but may be available from the corresponding author upon reasonable request.

Code Availability Not applicable.

Authors Contributions JLG designed the study, provided oversight of the data analysis, and drafted the manuscript. TDG assisted with the study design, conducted all analyses, and contributed significantly to the manuscript writing and editing. SFB provided general oversight of the ASI-MV program and made significant contributions to the manuscript. All authors read and approved the final version.

Open Access This article is licensed under a Creative Commons Attribution-NonCommercial 4.0 International License, which permits any non-commercial use, sharing, adaptation, distribution and reproduction in any medium or format, as long as you give appropriate credit to the original author(s) and the source, provide a link to the Creative 
Commons licence, and indicate if changes were made. The images or other third party material in this article are included in the article's Creative Commons licence, unless indicated otherwise in a credit line to the material. If material is not included in the article's Creative Commons licence and your intended use is not permitted by statutory regulation or exceeds the permitted use, you will need to obtain permission directly from the copyright holder. To view a copy of this licence, visit http://creativecommons.org/licenses/by-nc/4.0/.

\section{References}

1. Dal Pan GJ. Real-world data, advanced analytics, and the evolution of postmarket drug safety surveillance. Clin Pharmacol Ther. 2019;106(1):28-30.

2. Janssen Pharmaceuticals. ULTRAM $®$ (tramadol hydrochloride) Medication Guide. 2019. https://www.janssenlabels.com/packageinsert/product-monograph/prescribing-information/ULTRAM-pi. pdf. Accessed 30 Aug 2020

3. FDA. Orange Book: Approved Drug Products with Therapeutic Equivalence Evaluations. 2020. https://www.accessdata.fda.gov/ Scripts/cder/ob/search_product.cfm. Accessed 30 Aug 2020.

4. Department of Justice, Drug Enforcement Administration. Schedules of Controlled Substances: Placement of Tramadol into Schedule IV; Final Rule. Federal Register vol 79, no 127, 2 Jul 2014. 2020. https ://www.deadiversion.usdoj.gov/fed_regs/rules/2014/fr0702.htm. Accessed 30 Aug 2020.

5. Drug Enforcement Agency. Definition of Controlled Substances Schedules. 2020. https://www.deadiversion.usdoj.gov/sched ules/\#define. Accessed 30 Aug 2020.

6. Thiels CA, Habermann EB, Hooten WM, Jeffery MM. Chronic use of tramadol after acute pain episode: cohort study. BMJ. 2019. https ://doi.org/10.1136/bmj.11849.

7. Harrison ML, Walsh TL. The effect of a more strict 2014 DEA schedule designation for hydrocodone products on opioid prescription rates in the United States. Clin Toxicol. 2019;57(11):1064-72.

8. Jeong S, Tchoe HJ, Li J, Shin JY. All-cause mortality associated with tramadol use: a case-crossover study. Drug Saf. 2019;42(6):785-96.

9. Zeng C, Dubreuil M, LaRochelle MR, Lu NA, Wei J, Choi HK, Lei G, Zhang Y. Association of tramadol with all-cause mortality among patients with osteoarthritis. JAMA. 2019;321(10):969-82.

10. Taghipour DJ, Carone MA, Wolfe SM. Citizen petition to the DEA and FDA on tramadol. 2020. https://www.citizen.org/wp-content/ uploads/2497.pdf?eType=EmailBlastContent\&eId=f75d6700-4f5446af-97bd-4d025e1c1fdf. Accessed 30 Aug 2020.

11. World Health Organization, Expert Committee on Drug Dependence. Tramadol Update Review Report, Agenda item 6.1. 2020. https://www.who.int/medicines/areas/quality_safety/6_1_Updat e.pdf. Accessed 30 Aug 2020.

12. Dunn KE, Bergeria CL, Huhn AS, Strain E. A systematic review of laboratory evidence for the abuse potential of tramadol in humans. Front Psychiatry. 2019;10:704. https://doi.org/10.3389/fpsyt 2019.00704.

13. Smart R, Kase CA, Taylor EA, Lumsden S, Smith SR, Stein BD. Strengths and weaknesses of existing data sources to support research to address the opioids crisis. Prev Med Rep. 2020;17:101015. https://doi.org/10.1016/j.pmedr.2019.101015.
14. Vosburg SK, Beaumont J, Dailey-Govoni T, Butler SF, Green JL. Evaluation of abuse and route of administration of extended-release tapentadol among treatment-seeking individuals, as captured by the Addiction Severity Index-Multimedia Version (ASI-MV). Pain Med. 2019. https://doi.org/10.1093/pm/pnz250.

15. Butler SF, Black RA, Severtson SG, Dart RC, Green JL. Understanding abuse of buprenorphine/naloxone film versus tablet products using data from ASI-MV® substance use disorder treatment centers and RADARS $®$ System Poison Centers. J Subst Abuse Treat. 2017;84:42-9.

16. Butler SF, McNaughton EC, Black RA. Tapentadol abuse potential: a postmarketing evaluation using a sample of individuals evaluated for substance abuse treatment. Pain Med. 2015;16(1):119-30.

17. Cassidy TA, DasMahapatra P, Black RA, Wieman MS, Butler SF. Changes in prevalence of prescription opioid abuse after introduction of an abuse-deterrent opioid formulation. Pain Med. 2014;15:440-51.

18. Butler SF, Newman FL, Cacciola JS, Frank A, Budman SH, McLellan AT, Ford S, Blaine J, Gastfriend D, Moras K, Sallom IM, Barber JP. Predicting Addiction Severity Index (ASI) interviewer severity ratings for a computer-administered ASI. Psychol Assess. 1998;10(4):399-407.

19. Butler SF, Budman SH, Goldman RJ, Newman FL, Beckley KE, Trottier D, Cacciola JS. Initial validation of a computer-administered Addiction Severity Index: the ASI-MV. Psychol Addict Behav. 2001;15(1):4-12.

20. Vosburg SK, Severtson SG, Dart RC, Cicero TJ, Kurtz SP, Parrino MW, Green JL. Assessment of tapentadol API abuse liability with the researched abuse, diversion and addiction-related surveillance (RADARS) System. J Pain. 2018;19(4):439-53.

21. Murphy DL, Lebin JA, Severtson SG, Olsen HA, Dasgupta N, Dart RC. Comparative rates of mortality and serious adverse effects among commonly prescribed opioid analgesics. Drug Saf. 2018;41(8):787-95.

22. Wiegand TJ, Le Lait MC, Bartelson BB, Dart RC, Green JL. Analysis of the abuse and diversion of the buprenorphine transdermal delivery system. J Pain. 2016;17(6):745-52.

23. Dart RC, Cicero TJ, Surratt HL, Rosenblum A, Bartelson BB, Adams EH. Assessment of the abuse of tapentadol immediate release: the first 24 months. J Opioid Manag. 2012;8(6):395-402.

24. Wang KH, Fiellin DA, Becker WC. Source of prescription drugs used nonmedically in rural and urban populations. Am J Drug Alcohol Abuse. 2014;40(4):292-303.

25. Green JL, Bucher Bartelson B, Le Lait MC, Roland CL, Masters ET, Mardekian J, Bailey E, Dart RC. Medical outcomes associated with prescription opioid abuse via oral and non-oral routes of administration. Drug Alcoh Depend. 2017;175:140-5.

26. Cicero TJ, Ellis MS, Kasper ZA. Polysubstance use: a broader understanding of substance use during the opioid crisis. Am J Public Health. 2020;110:244-50.

27. Secora A, Trinidad JP, Zhang R, Gill R, Dal Pan G. Drug availability adjustments in population-based studies of prescription opioid abuse. Pharmacoepidemiol Drug Saf. 2017;26(2):180-91. 